\begin{tabular}{ll}
\hline \hline MINING AND METALLURGY INSTITUTE BOR & ISSN: 2334-8836 \\
& UDK: 622 \\
\hline \hline
\end{tabular}

Ljubinko Savić $^{*}$, Nebojša Vidanović ${ }^{* *}$, Rade Tokalic $^{* *}$

\title{
STUDYING THE EFFECTS OF BOREHOLE LENGTH ON THE IMPACT DRILLING SPEED IN DIFFERENT ROCKS
}

\begin{abstract}
The performed tests in this work include a wide range of rock masses of the Kopaonik massive of different strength $(f=2.3$ - 16). Drilling was done with drill hammers VK-24 and RK-21 at optimum sharpening angle with different drill rod lengths (0.8, 1.2, 1.6, 2.4 and 3.2). Compressed air pressure is $0.60 \mathrm{MPa}$ and diameter of drilling chisel $d=32 \mathrm{~mm}$. The obtained results and analysis indicate that it is possible to determine the interdependence between the borehole length and drilling speed. The results of these tests are shown in Tables 1, 2, 3 and 4 and diagrams 1, 2, 3 and 4.
\end{abstract}

Keywords: drill hammer, drill chisel, drilling speed, rock massive, borehole length

\section{INTRODUCTION}

Drilling speed is also the function of drilling length because the energy losses are increased by the increase of borehole length during transmission over more and longer drill rod. The increase of drill rod length causes the increase of its mass, which makes the process of turning more difficult, and, as a direct consequence, the piston moves back slowly, what extends the time of one cycle, and therefore has an impact on the number of strikes. All of this has an impact on the extent of rock mass destruction at the borehole head, and thus on drilling speed, expressed in some value of units.

The relation between the borehole length and drilling speed is not so simple and depends on many factors but it is possible to establish a way in which these relations move using the experimental forms. For example, according to the research, in the former Soviet Union, carried out by the Institute Vugi it is possible to use the empirical form:

$$
\mathrm{V}=\mathrm{Vo} \cdot(1.06-0.04 \cdot \ell)
$$

Where:

$\mathrm{V}$ - speed that is required if the borehole length is $\ell$,

Vo - speed developed at $1.5 \mathrm{~m}$. This speed is often taken as the initial drilling speed.

The current research in this field shows that it is the most reliably to perform the experimental research on the basis of the results, for each operating environment and operation mode, to determine correlations between changes in speed in relation to the change in borehole length and change of hammer drill. It can be seen from the above mentioned what is the impact of borehole length on drilling speed.

\section{CONDITIONS OF CARRIED OUT TESTS}

The tests were carried out in different areas of the rock massif Kopaonik. The rocks and minerals of different strength

\footnotetext{
* University of Pristina, Faculty of Technical Science, K. Mitrovica, ljsavic@open.telekom.rs

** University of Belgrade, Faculty of Mining and Geology,vidanovic@rgf.ac.rs, tokalic@rgf.ac.rs
} 
( $f=2.3-16)$ were tested. Drilling of boreholes was performed by drill hammers VK-24 and RK-21 with diameter of drill bit blade $\mathrm{d}=32 \mathrm{~mm}$ and the air pressure $0.6 \mathrm{MPa}$. The standard drill rods (SANDVIK COROMANT), length $(0.8,1.2,1.6$, 2.4, and 3.2) were adopted for testing.

\section{ANALYSIS OF THE OBTAINED RESULTS}

Based on the results for each drill hammer and every working environment, the dependences between the drilling speed and borehole length are calculated. Based on the results from the working environments, the integral dependences are given for each parameter and corresponding drill hammer. The used data for this calculation and their graphical and analytical interpretations are presented in Tables 1 and 2 and diagrams 1 and 2.

\section{Drilling speed depending on drill chisel length for weak rocks $(\mathbf{f}<5)$}

\section{Table 1.}

\begin{tabular}{|c|c|c|c|c|c|c|c|c|c|}
\hline $\begin{array}{c}\text { Chisel } \\
\text { length }\end{array}$ & \multicolumn{7}{|c|}{ Drilling speed per working environments for drill hammer $\boldsymbol{R K}-\mathbf{2 1}$} \\
\cline { 2 - 9 } $\boldsymbol{l}(\boldsymbol{m})$ & $\mathbf{1 2}$ & $\mathbf{1 0}$ & $\mathbf{1 1}$ & $\mathbf{1 3}$ & $\mathbf{1}$ & $\mathbf{4}$ & $\mathbf{2}$ & $\mathbf{6}$ & $\mathbf{9}$ \\
\hline 0.80 & 53.80 & 36.80 & 48.40 & 51.00 & 40.80 & 50.80 & 42.80 & 47.20 & 31.80 \\
1.20 & 52.80 & 36.40 & 47.20 & 49.80 & 40.00 & 49.60 & 42.00 & 46.40 & 30.80 \\
1.60 & 52.50 & 35.20 & 46.80 & 49.30 & 39.60 & 49.20 & 41.40 & 45.60 & 30.40 \\
2.40 & 50.60 & 34.00 & 45.20 & 47.60 & 38.40 & 47.60 & 39.80 & 44.00 & 29.60 \\
3.20 & 49.00 & 32.60 & 43.80 & 45.40 & 36.80 & 45.90 & 38.60 & 42.60 & 28.40 \\
\hline
\end{tabular}

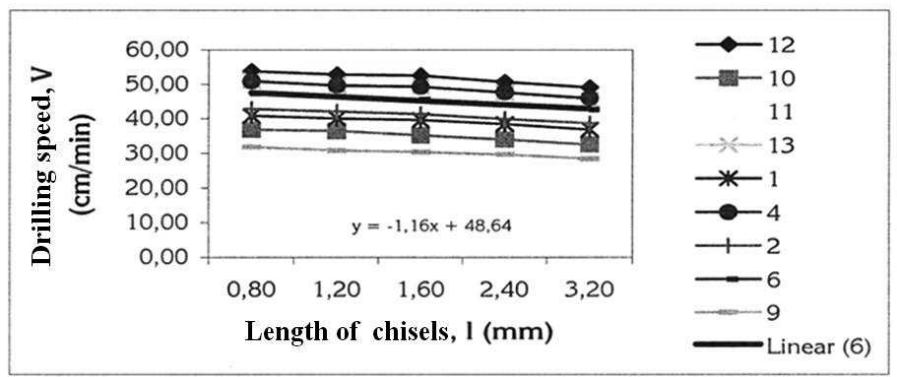

Figure 1 a Diagram of dependence between drilling speed and drill chisel length in drilling by drill hammer $R K-21$, the air pressure $p=0.60 \mathrm{MPa}$ and drill chisel diameter $d=32 \mathrm{~mm}$. Drilling speed depending on drill chisel length for weak rocks $(f<5)$.

\section{Drilling speed depending on drill chisel} length for hard rocks $(f>5)$

Continuation of Table 1

\begin{tabular}{|c|c|c|c|c|c|c|c|c|}
\hline \multirow{2}{*}{$\begin{array}{c}\text { Chisel } \\
\text { length }\end{array}$} & \multicolumn{7}{|c|}{ Drilling speed per working environments for drill hammer $\boldsymbol{R K}-\mathbf{2 1}$} \\
\cline { 2 - 9 } $\boldsymbol{l}(\boldsymbol{m})$ & $\mathbf{7}$ & $\mathbf{5}$ & $\mathbf{3}$ & $\mathbf{1 6}$ & $\mathbf{1 5}$ & $\mathbf{1 7}$ & $\mathbf{1 4}$ & $\mathbf{8}$ \\
\hline 0.80 & 36.80 & 39.20 & 32.00 & 38.80 & 33.70 & 24.20 & 27.00 & 21.00 \\
1.20 & 36.40 & 38.40 & 31.40 & 38.00 & 33.00 & 24.80 & 26.50 & 20.80 \\
1.60 & 35.30 & 37.80 & 30.60 & 37.50 & 32.70 & 23.50 & 26.30 & 20.30 \\
2.40 & 33.90 & 36.60 & 29.80 & 36.40 & 31.40 & 22.70 & 25.40 & 19.40 \\
3.20 & 32.80 & 35.80 & 28.60 & 35.20 & 30.50 & 21.50 & 24.20 & 18.60 \\
\hline
\end{tabular}




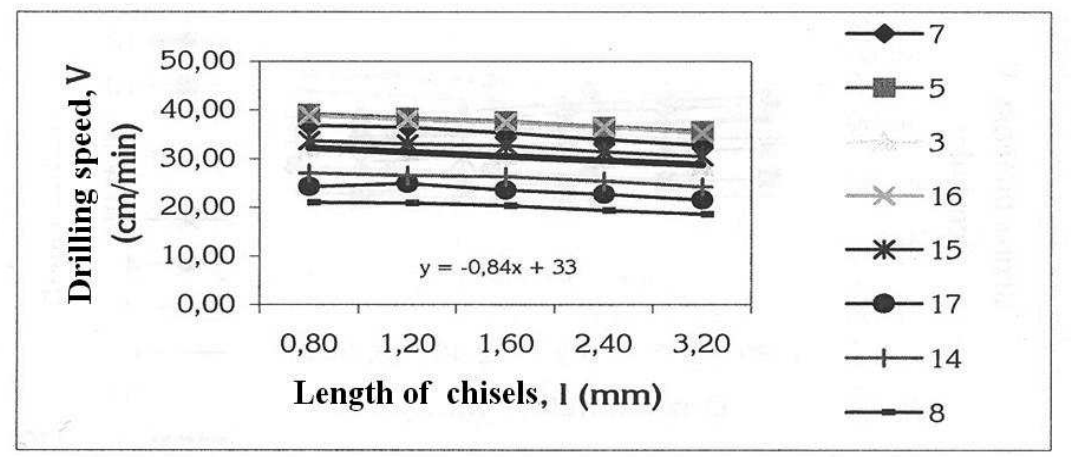

Figure 1 b Diagram of dependence between drilling speed and drill chisel length in drilling by drill hammer $R K-21$, the air pressure of $p=0.60 \mathrm{MPa}$ and drill chisel diameter $d=32 \mathrm{~mm}$. Drilling speed depending on drill chisel length for hard rocks $(f>5)$

Drilling speed depending on drill chisel length for weak rocks $(\mathbf{f}<\mathbf{5})$

Table 2

\begin{tabular}{|c|c|c|c|c|c|c|c|c|c|}
\hline \multirow{2}{*}{$\begin{array}{c}\text { Chisel } \\
\text { length }\end{array}$} & \multicolumn{8}{|c|}{ Drilling speed per working environments for drill hammer VK-24 } \\
\cline { 2 - 10 } $\boldsymbol{l}(\boldsymbol{m})$ & $\mathbf{1 2}$ & $\mathbf{1 0}$ & $\mathbf{1 1}$ & $\mathbf{1 3}$ & $\mathbf{1}$ & $\mathbf{4}$ & $\mathbf{2}$ & $\mathbf{6}$ & $\mathbf{9}$ \\
\hline 0.80 & 67.80 & 42.60 & 55.80 & 65.00 & 52.00 & 65.40 & 55.20 & 60.60 & 37.20 \\
1.20 & 66.80 & 41.40 & 54.60 & 64.20 & 50.80 & 64.00 & 54.60 & 59.80 & 36.60 \\
1.60 & 65.40 & 40.80 & 53.80 & 62.40 & 50.20 & 62.80 & 53.40 & 58.40 & 35.80 \\
2.40 & 63.20 & 39.60 & 52.20 & 60.80 & 48.80 & 60.20 & 51.80 & 56.00 & 34.60 \\
3.20 & 61.40 & 37.80 & 50.60 & 58.60 & 47.20 & 58.20 & 50.00 & 54.60 & 33.20 \\
\hline
\end{tabular}

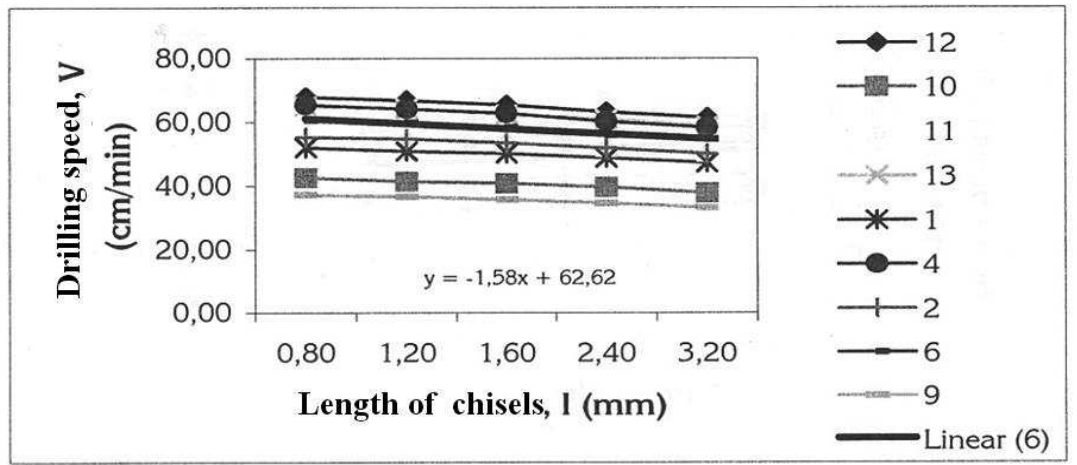

Figure 2 a Diagram of dependence between drilling speed and drill chisel length in drilling by drill hammer VK -24, the air pressure $p=0.60 \mathrm{Mpa}$ and drill chisel diameter $d=32 \mathrm{~mm}$. Drilling speed depending on drill chisel length for weak rocks $(f<5)$ 


\section{Drilling speed depending on drill chisel length for hard rocks $(\mathbf{f}>5)$}

Continuation of Table 2

\begin{tabular}{|c|c|c|c|c|c|c|c|c|}
\hline \multirow{2}{*}{$\begin{array}{c}\text { Chisel } \\
\text { length }\end{array}$} & \multicolumn{7}{|c|}{ Drilling speed in working environments for drill hammers $V K-24$} \\
$\boldsymbol{l}(\boldsymbol{m})$ & $\mathbf{7}$ & $\mathbf{5}$ & $\mathbf{3}$ & $\mathbf{1 6}$ & $\mathbf{1 5}$ & $\mathbf{1 7}$ & $\mathbf{1 4}$ & $\mathbf{8}$ \\
\cline { 2 - 9 } & 47.10 & 46.60 & 37.90 & 43.20 & 37.60 & 28.80 & 33.60 & 23.80 \\
0.80 & 46.80 & 45.80 & 37.50 & 42.80 & 37.00 & 28.40 & 33.00 & 23.00 \\
1.20 & 45.60 & 45.10 & 36.80 & 42.30 & 36.80 & 28.00 & 32.50 & 22.50 \\
1.60 & 44.20 & 43.80 & 35.20 & 40.60 & 35.60 & 27.20 & 31.20 & 21.40 \\
2.40 & 42.80 & 42.20 & 33.80 & 39.00 & 34.40 & 26.00 & 30.00 & 20.40 \\
3.20 & &
\end{tabular}

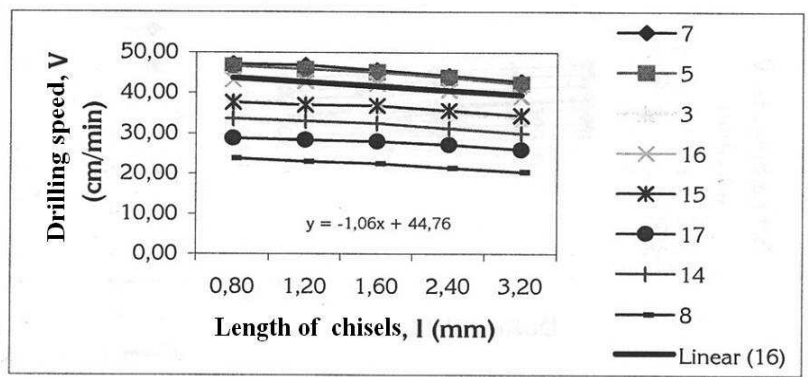

Figure $2 \mathbf{b}$ Diagram of dependence between drilling speed and drill chisel length in drilling by drill hammer VK-24, the air pressure $p=0.60 \mathrm{MPa}$ and drill chisel diameter $d=32 \mathrm{~mm}$. Drilling speed depending on drill chisel length for hard rocks $(f>5)$

\section{CONCLUSION}

With increasing borehole length, the need for longer length of drilling chisels increases, which also implies higher weight. All of these increase the resistance to the drilling chisel rotation, makes difficult cleaning the borehole and increase the energy transfer losses from piston to the drill crown blade. These resistances and losses directly impact the drilling speed showing a tendency to decrease after increasing the drilling length. Based on the obtained results, it can be concluded that dispersal of data, in an integrated setting of all tested environments is high what is the reason of a large range of mechanical properties of tested environments, and changing their changeable behavior when it comes to the resistance on drilling.

The obtained results and their analysis indicate that it is possible to determine the interdependences between the above mentioned parameters.

\section{REFERENCES}

[1] Lj. Savić, The Effect of the Most Important Parameters of Chisel in Impact Drilling on the Speed of Borehole Development for Conditions in the Mines Zuta Prla, RGF Belgrade, 1990;

[2] P. Jovanović, Development the Mining Workings, RGF Belgrade, 1990;

[3] Lj. Savić, The Effect of Technological Parameters on the Speed of Rotary Impact Drilling in Hard Rocks, RGF Belgrade, 2003;

[4] P. Jovanović, Mechanical Procedures of Rock Destruction During Mining Operations RGF Belgrade, 1980;

[5] Brochures of Drilling Rig Manufacturers;

[6] M. A. Kobiliska, General Mining Operations, Gradjevinska knjiga 


\begin{tabular}{ll}
\hline \hline INSTITUT ZA RUDARSTVO I METALURGIJU BOR & ISSN: 2334-8836 \\
& UDK: 622 \\
\hline \hline
\end{tabular}

Ljubinko Savić, Nebojša Vidanovic ${ }^{* *}$, Rade Tokalic ${ }^{* *}$

\title{
PROUČAVANJE UTICAJA DUŽINE BUŠOTINE NA BRZINU UDARNOG BUŠENJA U RAZLIČITIM STENAMA
}

\begin{abstract}
Izvod
Ispitivanja koja su izvršena u okviru ovog rada obuhvataju široku lepezu stenskih masa Kopaoničkog masivva različite čvrstoće ( $f=2,3$ - 16). Bušenje je vršeno sa bušaćim čekićima VK-24 i RK-21, sa optimalnim uglom oštrenja sa različitim dužinama bušaće šipke (0.8, 1.2, 1.6, 2.4 i 3.2). Pritisak sabijskog vazduha 0,60 MPa, prečnik bušećeg dleta $d=32 \mathrm{~mm}$. Dobijeni rezultati $i$ njihova analiza ukazuju da je moguće utvrditi međusobne zavisnosti između dužine bušotine i brzine bušenja. Dobijeni rezultati ovih istraživanja prikazani su u tabelama1, 2, 3 i 4 i diijagrama 1, 2, 3 i 4.
\end{abstract}

Ključne reči: bušaći čekić, bušeće dleto, brzina bušenja, stenska sredina, dužina bušotine

\section{UVOD}

Brzina bušenja funkcija je i dužine bušotine, jer sa povećanjem dužine bušotine povećavaju se gubici energije prilikom prenosa kroz sve dužu i dužu bušaću šipku. Isto tako sa povećanjem dužine šipke bušećeg dleta povećava se i njena masa, što otežava proces zaokretanja, a kao direktna posledica javlja se sporije kretanje klipa unazad, što produžava vreme jednog ciklusa, pa samim tim ima uticaja i na broj udara. Sve ovo zajedno ima svog uticaja na obim razaranja stenske mase na čelu bušotine, pa samim tim i na brzinu bušenja izraženu kroz neku od vrednosnih jedninica.

Zavisnost između dužine bušotine i brzine bušenja nije tako jednostavno i zavisi od mnogih činilaca ali je pomoću eksperimentalnih obrazaca moguće ustanoviti relaciju u kojima se ovi odnosi kreću. Tako na primer, prema istraživanjima u bivšem Sovjetskom Savezu izvršeni od strane Instituta Vugi kod ovih proučavanja moguće je korisititi empirijski obrazac:

$$
\mathrm{V}=\mathrm{Vo}(1,06-0,04 \cdot \ell)
$$

gde su:

V - brzina koja se traži ako je dužina bušotine, $\ell$,

Vo - brzina koja se postiže od 1,5 m. Ova brzina se često uzima i kao početna brzina bušenja.

Aktuelna istraživanja $u$ ovoj oblasti pokazuju da je najpouzdanije izvršiti ogledna istraživanja i na osnovu rezultata, za svaku radnu sredinu i režim rada ustanoviti korelacione odnose između promene brzine u odnosu na promenu dužine bušotine i na promenu bušaćeg čekića. Iz napred izloženog se vidi od kolikog je uticaja dužina bušotine na brzinu bušenja.

\section{USLOVI POD KOJIMA SU IZVRŠENA ISTRAŽIVANJA}

Istraživanja su obavljena u različitim stenskim sredinama Kopaoničkog masiva. Ispitivane su stene i rude različite čvrstoće

\footnotetext{
*Univerzitet u Prištini, Fakultet tehničkih nauka, K. Mitrovica, ljsavic@open.telekom.rs

** Univerzitet u Beogradu, Rudarsko-geološkifakultet, vidanovic@rgf.ac.rs, tokalic@rgf.ac.rs
} 
( $f=2.3-16)$. Bušenje minskih bušotina vršeno je bušaćim čekićima VK-24 i RK-21, sa prečnikom sečiva bušaće krune $d=32 \mathrm{~mm}$, sa pritiskom vazduha 0,6 MPpa.

$\mathrm{Za}$ istraživanje usvojene su standardne bušaće šipke (SANDVIK COROMANT) DUŽINE $(0.8,1.2,1.6,2.4$ i 3.2).

\section{ANALIZA DOBIJENIH REZULTATA}

$\mathrm{Na}$ osnovu dobijenih rezultata za svaki bušeći čekić i svaku radnu sredinu proraču- nate zavisnosti odnosa između brzine bušenja i dužine bušotine. Na osnovu pojedinačnih rezultata po radnim sredinama, za svaki parametar i odgovarajući bušaći čekić, date su integralne zavisnosti. Podaci koji su poslužili za ovaj proračun i njihove grafičke i analitičke interpretacije prikazane su u tabelama 1 i 2 i na dijagramima 1 i 2.

\section{Brzina bušenja u zavisnosti od dužine} bušaćeg dleta za slabe stene $(\mathbf{f}<5)$

Tabela 1.

\begin{tabular}{|c|c|c|c|c|c|c|c|c|c|c|}
\hline \multirow{2}{*}{$\begin{array}{c}\text { Dužina } \\
\text { dleta }\end{array}$} & \multicolumn{7}{|c|}{ Brzina bušenja po radnim sredinama za bušaći čekić $\boldsymbol{R} \boldsymbol{K}$-21 } \\
$\boldsymbol{l} \boldsymbol{l}(\boldsymbol{m})$ & $\mathbf{1 2}$ & $\mathbf{1 0}$ & $\mathbf{1 1}$ & $\mathbf{1 3}$ & $\mathbf{1}$ & $\mathbf{4}$ & $\mathbf{2}$ & $\mathbf{6}$ & $\mathbf{9}$ \\
\hline 0,80 & 53,80 & 36,80 & 48,40 & 51,00 & 40,80 & 50,80 & 42,80 & 47,20 & 31,80 \\
1,20 & 52,80 & 36,40 & 47,20 & 49,80 & 40,00 & 49,60 & 42,00 & 46,40 & 30,80 \\
1,60 & 52,50 & 35,20 & 46,80 & 49,30 & 39,60 & 49,20 & 41,40 & 45,60 & 30,40 \\
2,40 & 50,60 & 34,00 & 45,20 & 47,60 & 38,40 & 47,60 & 39,80 & 44,00 & 29,60 \\
3,20 & 49,00 & 32,60 & 43,80 & 45,40 & 36,80 & 45,90 & 38,60 & 42,60 & 28,40 \\
\hline
\end{tabular}

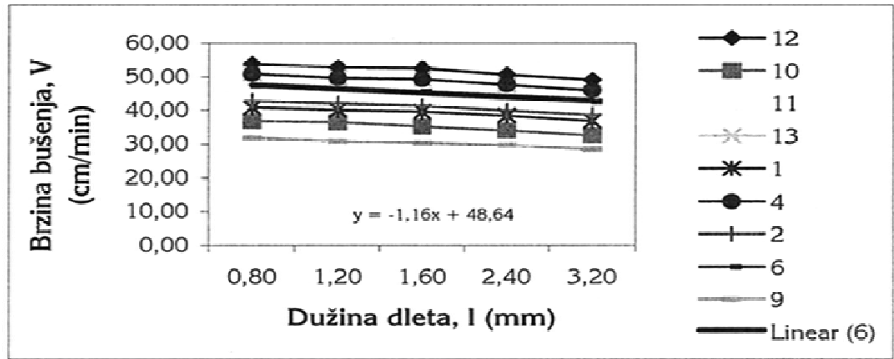

Sl. 1 a. Dijagram zavisnosti brzine bušenja od dužine bušaćeg dleta kod bušenja bušaćim čekićem $R K-21$, pritisak vazduha od $p=0,60 \mathrm{MPa}$ i prečnikom bušaćeg dleta $d=32 \mathrm{~mm}$ Brzina bušenja u zavisnosti od dužine bušaćeg dleta za slabe stene $(f<5)$

Brzina bušenja u zavisnosti od dužine bušaćeg dleta za čvrste stene (f $>5$ )

Nastavak tabele 1

\begin{tabular}{|c|c|c|c|c|c|c|c|c|}
\hline $\begin{array}{c}\text { Dužina } \\
\text { dleta }\end{array}$ & \multicolumn{7}{|c|}{ Brzina bušenja po radnim sredinama za bušaći čekić $\boldsymbol{R K}-21$} \\
\cline { 2 - 9 } $\boldsymbol{l}(\boldsymbol{m})$ & $\mathbf{7}$ & $\mathbf{5}$ & $\mathbf{3}$ & $\mathbf{1 6}$ & $\mathbf{1 5}$ & $\mathbf{1 7}$ & $\mathbf{1 4}$ & $\mathbf{8}$ \\
\hline 0,80 & 36,80 & 39,20 & 32,00 & 38,80 & 33,70 & 24,20 & 27,00 & 21,00 \\
1,20 & 36,40 & 38,40 & 31,40 & 38,00 & 33,00 & 24,80 & 26,50 & 20,80 \\
1,60 & 35,30 & 37,80 & 30,60 & 37,50 & 32,70 & 23,50 & 26,30 & 20,30 \\
2,40 & 33,90 & 36,60 & 29,80 & 36,40 & 31,40 & 22,70 & 25,40 & 19,40 \\
3,20 & 32,80 & 35,80 & 28,60 & 35,20 & 30,50 & 21,50 & 24,20 & 18,60 \\
\hline
\end{tabular}




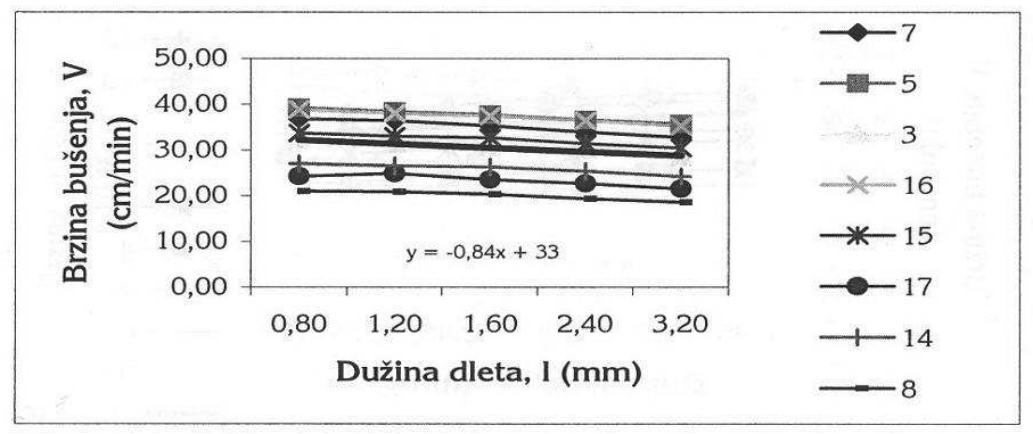

SI. 1 b. Dijagram zavisnosti brzine bušenja od dužine bušaćeg dleta kod bušenja bušaćim čekićem $R K-21$, pritisak vazduha od $p=0,60 \mathrm{MPa}$ i prečnikom bušaćeg dleta $d=32 \mathrm{~mm}$ Brzina bušenja u zavisnosti od dužine bušaćeg dleta za čvrste stene ( $f>5$ )

Brzina bušenja u zavisnosti od dužine bušaćeg dleta za slabe stene $(\mathbf{f}<5)$

Tabela 2.

\begin{tabular}{|c|c|c|c|c|c|c|c|c|c|}
\hline \multirow{2}{*}{$\begin{array}{c}\text { Dužina } \\
\text { dleta }\end{array}$} & \multicolumn{7}{|c|}{ Brzina bušenja po radnim sredinama za bušaći čekić VK-24 } \\
\cline { 2 - 10 } $\boldsymbol{l}(\boldsymbol{m})$ & $\mathbf{1 2}$ & $\mathbf{1 0}$ & $\mathbf{1 1}$ & $\mathbf{1 3}$ & $\mathbf{1}$ & $\mathbf{4}$ & $\mathbf{2}$ & $\mathbf{6}$ & $\mathbf{9}$ \\
\hline 0,80 & 67,80 & 42,60 & 55,80 & 65,00 & 52,00 & 65,40 & 55,20 & 60,60 & 37,20 \\
1,20 & 66,80 & 41,40 & 54,60 & 64,20 & 50,80 & 64,00 & 54,60 & 59,80 & 36,60 \\
1,60 & 65,40 & 40,80 & 53,80 & 62,40 & 50,20 & 62,80 & 53,40 & 58,40 & 35,80 \\
2,40 & 63,20 & 39,60 & 52,20 & 60,80 & 48,80 & 60,20 & 51,80 & 56,00 & 34,60 \\
3,20 & 61,40 & 37,80 & 50,60 & 58,60 & 47,20 & 58,20 & 50,00 & 54,60 & 33,20 \\
\hline
\end{tabular}

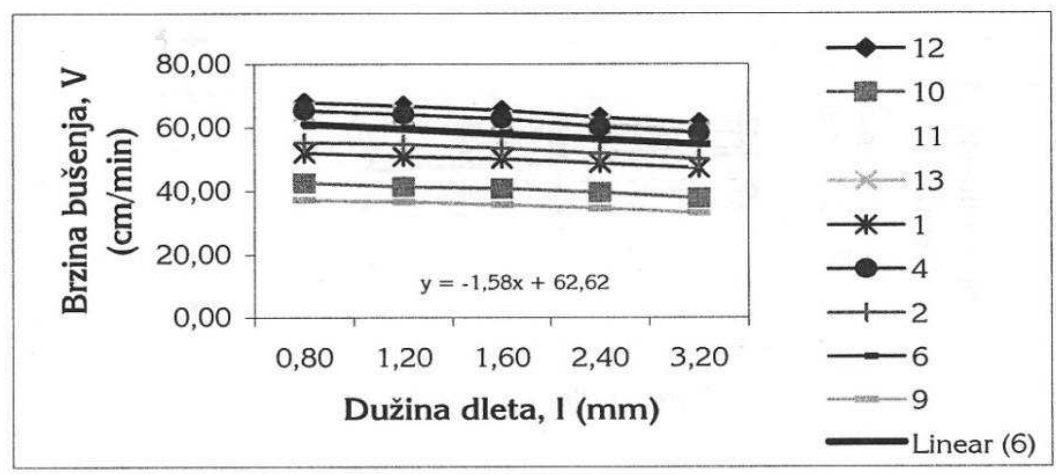

Sl. 2 a. Dijagram zavisnosti brzine bušenja od dužine bušaćeg dleta kod bušenja bušaćim čekićem $V K-24$, pritisak vazduha od $p=0,60 \mathrm{MPa}$ i prečnikom bušaćeg dleta $d=32 \mathrm{~mm}$ Brzina bušenja u zavisnosti od dužine bušaćeg dleta za slabe stene $(f<5)$ 


\section{Brzina bušenja u zavisnosti od dužine}

bušaćeg dleta za čvrste stene (f>5)

Nastavak tabele 2

\begin{tabular}{|c|c|c|c|c|c|c|c|c|}
\hline \multirow{2}{*}{$\begin{array}{c}\text { Dužina } \\
\text { dleta }\end{array}$} & \multicolumn{7}{|c|}{ Brzina bušenja po radnim sredinama za bušaći čekić $\boldsymbol{V K}$-24 } \\
\cline { 2 - 9 } $\boldsymbol{l}(\boldsymbol{m})$ & $\mathbf{7}$ & $\mathbf{5}$ & $\mathbf{3}$ & $\mathbf{1 6}$ & $\mathbf{1 5}$ & $\mathbf{1 7}$ & $\mathbf{1 4}$ & $\mathbf{8}$ \\
\hline 0,80 & 47,10 & 46,60 & 37,90 & 43,20 & 37,60 & 28,80 & 33,60 & 23,80 \\
1,20 & 46,80 & 45,80 & 37,50 & 42,80 & 37,00 & 28,40 & 33,00 & 23,00 \\
1,60 & 45,60 & 45,10 & 36,80 & 42,30 & 36,80 & 28,00 & 32,50 & 22,50 \\
2,40 & 44,20 & 43,80 & 35,20 & 40,60 & 35,60 & 27,20 & 31,20 & 21,40 \\
3,20 & 42,80 & 42,20 & 33,80 & 39,00 & 34,40 & 26,00 & 30,00 & 20,40 \\
\hline
\end{tabular}

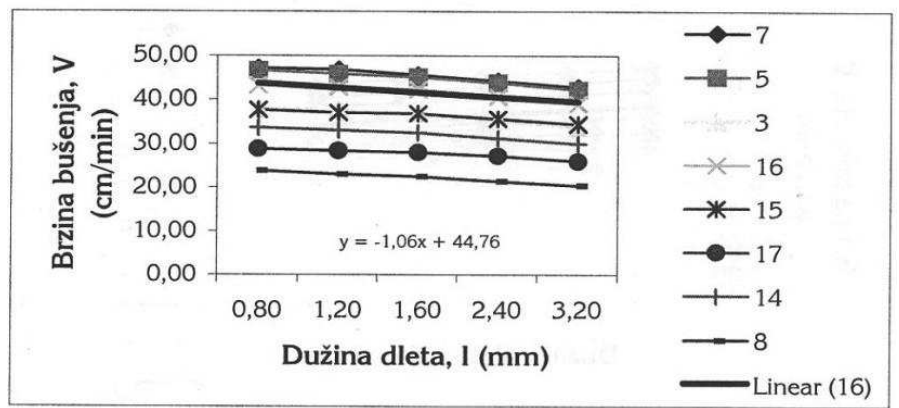

Sl. 2 b. Dijagram zavisnosti brzine bušenja od dužine bušaćeg dleta kod bušenja bušaćim čekićem $V K-24$, pritisak vazduha od $p=0,60 \mathrm{MPa}$ i prečnikom bušaćeg dleta $d=32 \mathrm{~mm}$ Brzina bušenja u zavisnosti od dužine bušaćeg dleta za čvrste stene ( $f>5)$

\section{ZAKLJUČAK}

Sa povećanjem dužine bušotine povećava se i potreba za većom dužinom bušećeg dleta, što za sobom povlači i veću njegovu težinu. Sve ovo povećava otpor prema obrtanju bušaćeg dleta, otežava čišćenje bušotine i uvećava gubitke prenosa energije od klipa do sečiva bušaće krune. Ovi otpori i gubici direktno utiču na brzinu bušenja, koja se povećanjem dužine bušenja pokazuje tendenciju pada. Na osnovu dobijenih rezultata moguće je zakljuciti da je osipanje podataka, kod integralnog postavljanja svih ispitivanih sredina, veliko što je razlog veliki raspon mehaničkih karakteristika ispitivanih sredina, i njihovo promenljivo ponašanje, kada je u pitanju otpor prema bušenju.

Dobijeni rezultat i njihova analiza ukazuju da je moguće utvrditi međusobne zavisnosti između napred navedenih parametara.

\section{LITERATURA}

[1] Lj. Savić, Uticaj najvažnijih parametara dleta kod udarnog bušenja na brzinu izrade minske bušotine za uslove rudnika Žuta Prla, RGF Beograd, 1990.

[2] P. Jovanović, Izrada jamskih prostorija, RGF Beograd, 1990.

[3] Lj. Savić, Uticaj tehnoloških parametara na brzinu udarno zaokretnog bušenja u čvrstim stenama, RGF Beograd, 2003.

[4] P. Jovanović, Mehanički postupci razaranja stena pri izvođenju rudarskih radova RGF, 1980.

[5] Prospekti proizvođača opreme za bušenje

[6] M. A. Kobiliška, Opšti rudarski radovi, Građevinska knjiga 